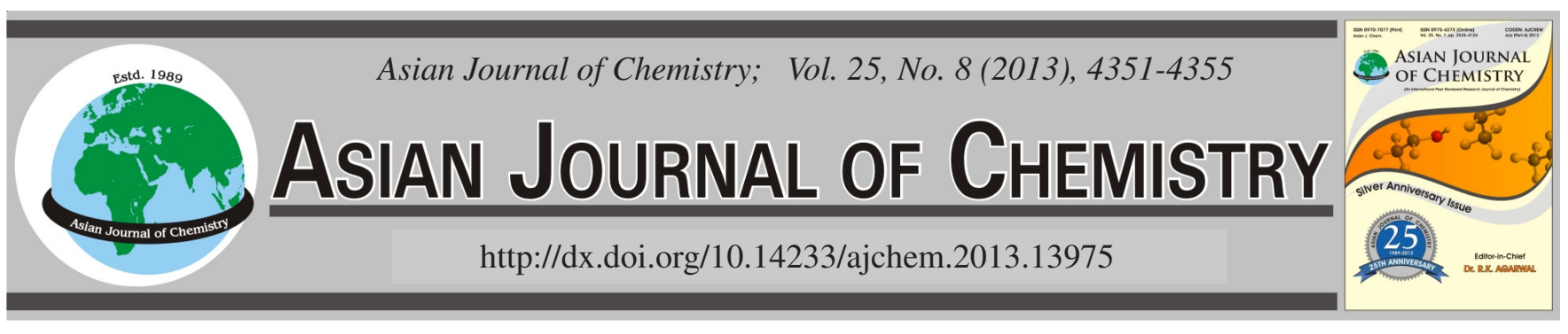

\title{
A New Method of Discrimination for Peaks of Enantiopure Compounds in Chiral Separation of HPLC
}

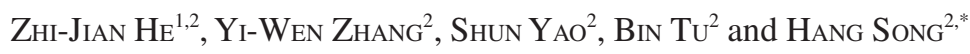

${ }^{1}$ College of Chemistry and Chemical Engineering, Mianyang Normal University, Mianyang, P.R. China

${ }^{2}$ Department of Pharmaceutical and Biological Engineering, Sichuan University, No. 24 South Section 1, Yihuan Road, Chengdu 610065, P.R. China

*Corresponding author: Tel/Fax: +86 28 85405221; E-mail: hangsong@ @scu.edu.cn; 376971478@qq.com; zyw734@gmail.com

\begin{abstract}
Diode array detector is one of the most popular detectors of high performance liquid chromatography (HPLC). With the full-band scanning, it has been used extensively for the qualitative and quantitative analysis of components. In this paper, a few groups of enantiomers, diastereomers, isomers and homologue were chosen as analytes and a new method of discrimination for their peaks in chromatogram of HPLC was tried to be developed. By using diode array detector, we obtained peak characteristics for them. By comparing chromatograms of enantiomers, diastereomers, isomers and homologue, we find that only the chromatograms of the enantiomers and diastereomers have considerable consistency. Then a new method of discrimination for peaks of enantiopure compounds by diode array detector has been established. This method can be used for research of chiral separation.
\end{abstract}

Key Words: High performance liquid chromatography, Diode array detector, Chiral separation, Enantiomer, Diastereomer, Isomer.

\section{INTRODUCTION}

In the recent years, research of chiral compounds developed very rapidly. A significant number of commercial available drugs are synthetic and chiral, therefore enantioseparation of chiral compounds has been attracting great interests ${ }^{1-3}$. Chromatographic methods have been used extensively in analysis, separation and purification of chiral compounds $s^{4-7}$. Separation of enantiomers can be achieved using different chromatographic techniques such as gas chromatography ${ }^{8}$, liquid chromatography ${ }^{9}$, supercritical fluid chromatography ${ }^{10}$ and capillary electrochromatography ${ }^{11}$. Among the previous methods, high performance liquid chromatography is well recognized as a powerful, fast, selective and highly efficient technique, which has been successfully employed for separation and determination of enantiomers of drugs ${ }^{12-14}$.

When we use HPLC for analyzing compound samples, we can choose appropriate detection system for different components of the molecular structure. For those compounds with UV absorption, they can be detected sensitively under the maximum absorption wavelength. Compared with traditional UV detection, diode array detector (DAD) can perform full-band scanning, which can be used for qualitative and quantitative analysis of components conveniently. Since Hewlett-Packard Company has introduced the first commercialization DAD in 1982. Diode array detector has obtained great development in many territories such as electronic technology and computer data processing and its applications are also constantly expanding at the same time. Today, diode array detector has become one of the most popular detectors in HPLC, which plays an important role in detection of natural products, medicine and other complex biological samples ${ }^{15-18}$.

In the preliminary research stage of chiral compounds, there is no single optical configuration of the sample in market, so it is impossible to use standard substance to determine peaks in HPLC. Therefore it brings much trouble to research of chiral resolution. In addition, Japan Jasco company has produce Jasco 2090 Plus optical rotation detector. It can be combined with HPLC as online optical rotation detector. When a pair of enantiomers enter the online optical rotation detector, a positive peak and a negative peak can be obtained ${ }^{19}$. So the optical rotation detector can also be used to discriminate chiral compounds. However, there are also some deficient for using optical rotation detector to discriminate chiral compounds. Some chiral compounds can not be detected by optical rotation detector for their small specific rotation. For example, when we separated racemate of 2-butylamine in our laboratory ${ }^{20}$, we used achiral reagent 3,5-dinitrobenzoic acid derivatize with it. Then the racemate of 2-butylamine derivatives have been separated successfully by Chiralcel OD-H column. However, when the racemate of 2-butylamine derivatives have been detected by optical rotation detector, we find that there was 
no corresponding positive and negative peaks. It indicated that the specific rotation of 2-butylamine with 3,5-dinitrobenzene acid derivative was so small that optical rotation detector can not detect it. So at this time optical rotation detector can not determine the peaks assignment of chiral compounds.

By using DAD, we can obtain three-dimensional chromatograms of absorption about time and wavelength, which can provide UV absorption information for analytes. In this paper, several groups of enantiomers, diastereomers, isomers and homologueas have been used as analytes, then the peak characteristics of above compounds detected by DAD have been obtained.

\section{EXPERIMENTAL}

Phenylethylamine, lactic acid, ibuprofen, (S)-2-methyl1-butanol and acenaphthene were purchased from Alfa Aesar (Tianjin, China). $n$-Hexane, ethanol and isopropanol of HPLC grade were supplied by Hangjia Chemical Co., Ltd. (Chengdu, China). EGCG, GCG, 4-methoxy benzoic acid, aniline, 8-hydroxyquinoline, benzylamine and 2-ethoxy-1-ethoxycarbonyl-1,2dihydroquinoline (EEDQ) were purchased from Sinopharm Chemical Reagent Co., Ltd (Chengdu, China). Other reagents supplied by Hangjia Chemical Co., Ltd. (Chengdu, China) were all in analytical level.

Analysis was carried out on a Shimadzu series liquid chromatography system, equipped with LC-20AT pump and SPD-M20A photodiode array detector (both from Shimadzu, Kyoto, Japan) and an Automatic Science (Tianjin, China) HCT360 LC column cooler/heater. Chromatographic parameters such as peak areas, retention times, theoretical plates, etc. were calculated using the Class-VP workstation (Shimadzu, Kyoto, Japan).

Chromatographic conditions: Chiralcel OD-H (250 mm $\times 4.6 \mathrm{~mm}$; particle size $5 \mu \mathrm{m}$ ) (Daicel, Japan), DNB-PG (250 $\mathrm{mm} \times 4.6 \mathrm{~mm}$; particle size $5 \mu \mathrm{m})$, DNB-Leu $(250 \mathrm{~mm} \times 4.6$ $\mathrm{mm}$; particle size $5 \mu \mathrm{m})$, Whelk-O1 $(250 \mathrm{~mm} \times 4.6 \mathrm{~mm}$; particle size $5 \mu \mathrm{m})$ (Regis Technologies, USA) and Kromasil CHI-DMB (250 mm $\times 4.6 \mathrm{~mm}$; particle size $5 \mu \mathrm{m})($ Akzo Nobel, Sweden) were used for the separation. Mobile phase consisted of $n$-hexane: isopropanol or ethanol (85:15 or other $\mathrm{v} / \mathrm{v}$ ) and the column temperature was at $25^{\circ} \mathrm{C}$. The flow rate

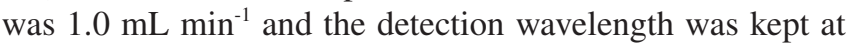
$254 \mathrm{~nm}$ or other. The injection volume was about $5 \mu \mathrm{L}$.

\section{RESULTS AND DISCUSSION}

Enantiomers: Two groups of enantiomers have been used for research of peak characteristics for enantiomers (derivatives of phenylethylamine and derivatives of lactic acid). The chromatograms of enantiomers were shown in Figs. 1 and 2, which indicated that the UV absorption for each enantiomer has considerable consistency.

Diastereomers: Two groups of diastereomers have been used for research of peak characteristics for diastereomers (derivatives of ibuprofen and EGCG/GCG). The chromatograms of enantiomers were shown in Figs. 3 and 4, which indicated that the UV absorption for each diastereomers has considerable consistency.
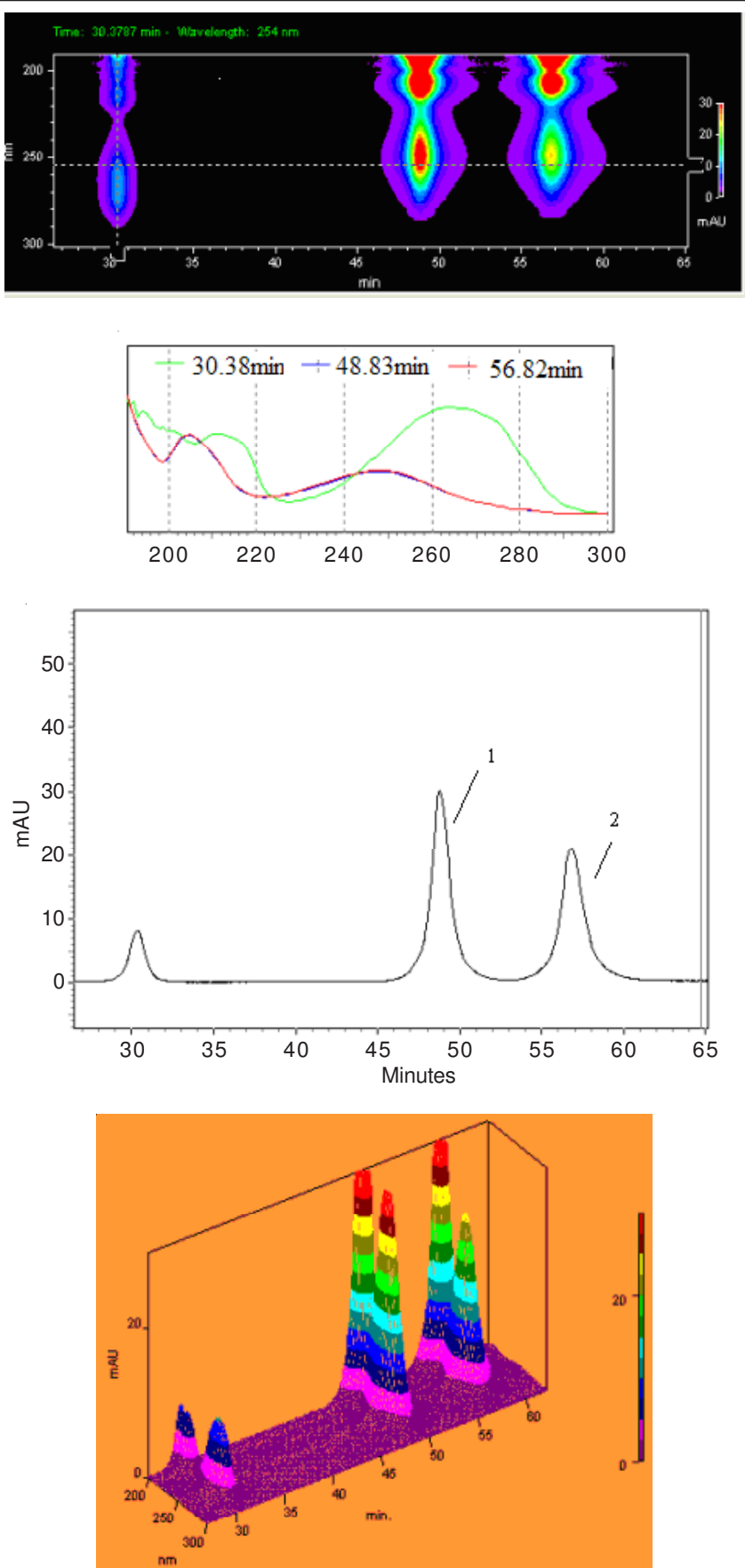

Fig. 1. Chromatograms of enantiomers (derivatives of phenylethylamine) $)^{21}$
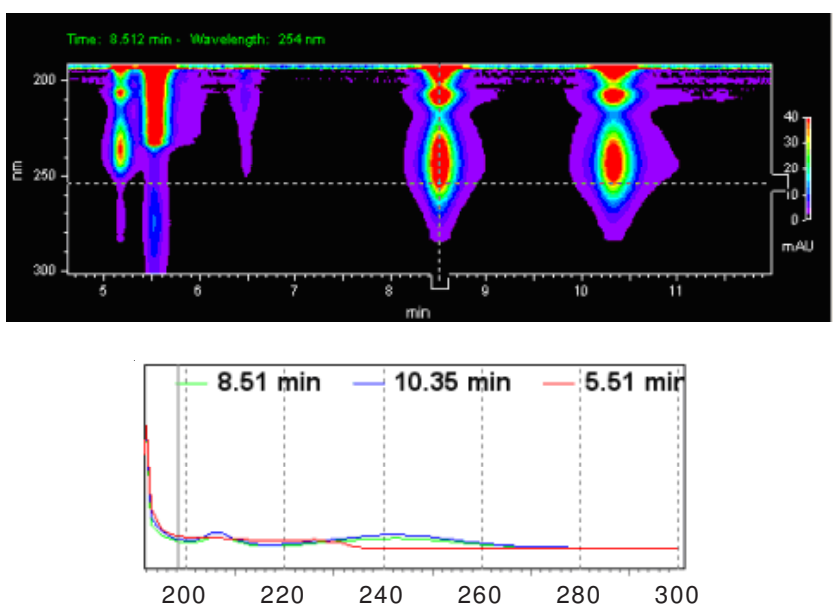

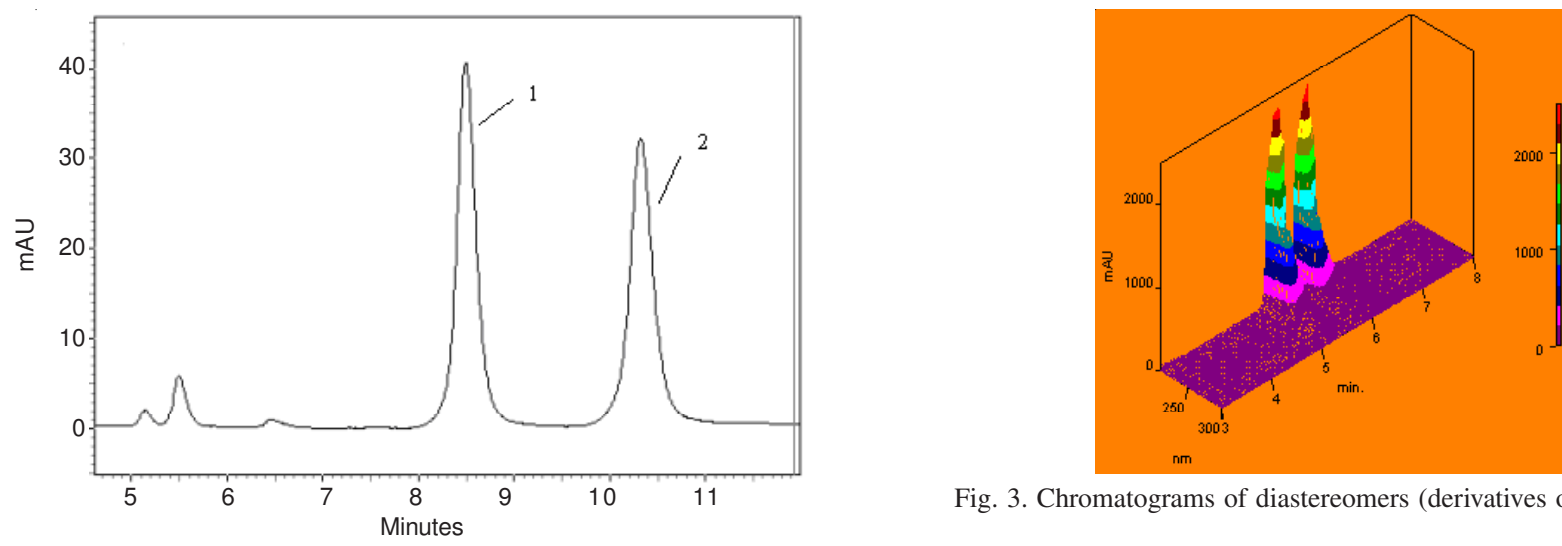

Fig. 3. Chromatograms of diastereomers (derivatives of ibuprofen)
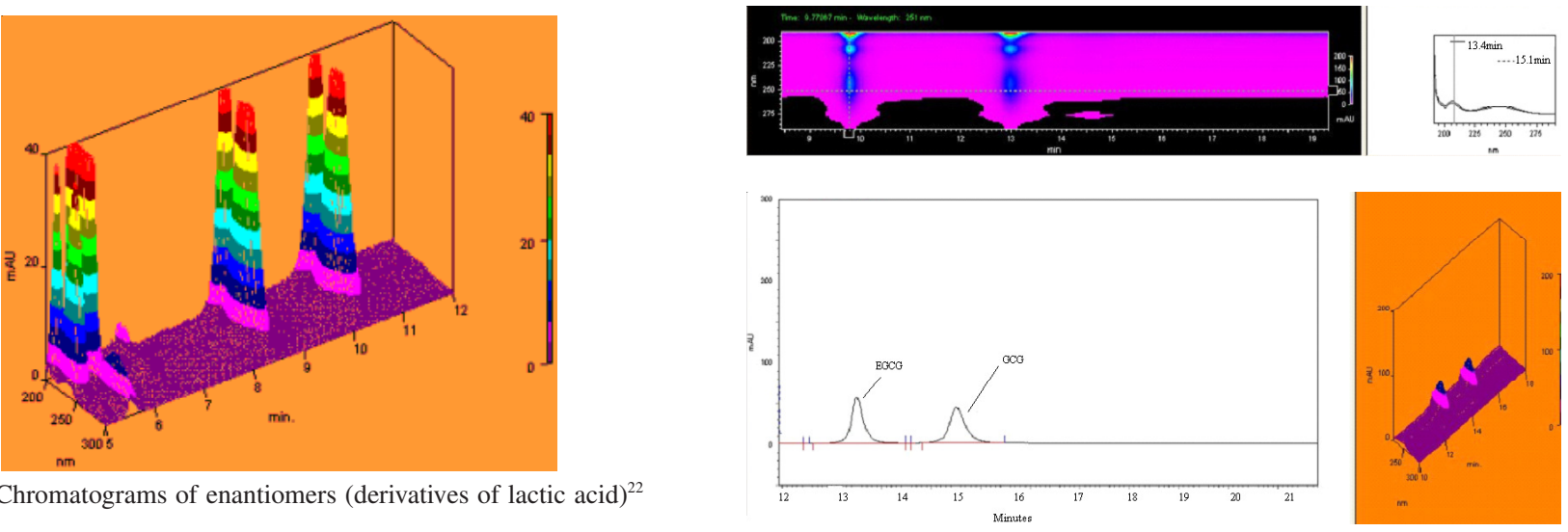

Fig. 2. Chromatograms of enantiomers (derivatives of lactic acid) ${ }^{22}$

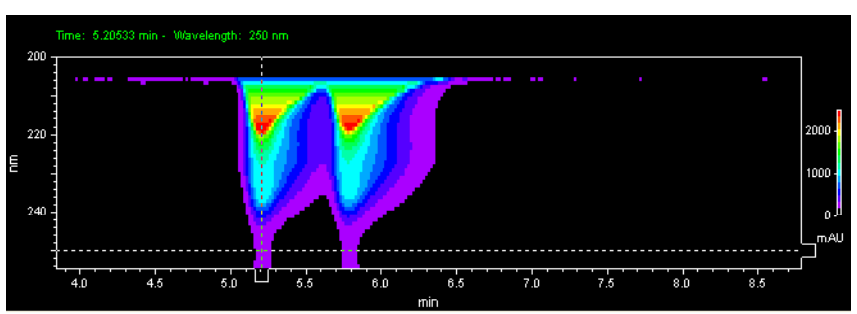

Fig. 4. Chromatograms of diastereomers (EGCG and GCG)

Isomers and homologueas: Three groups of isomers and homologueas have been used for research of peak characteristics. The chromatograms of enantiomers were shown in Fig. 5-7, which indicated that the UV absorption for each isomers and homologueas was significant differences.

Overlaid Spectra
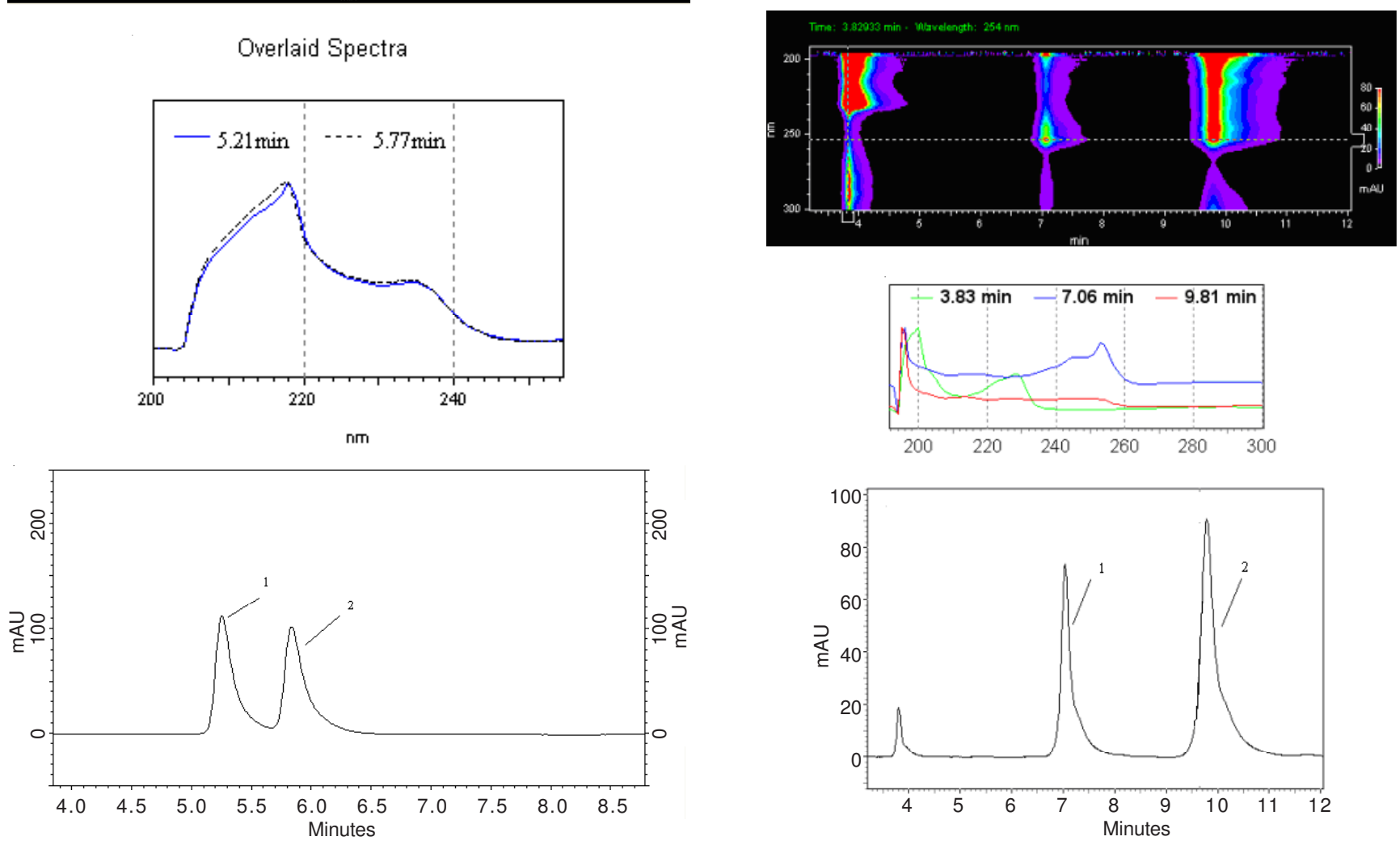


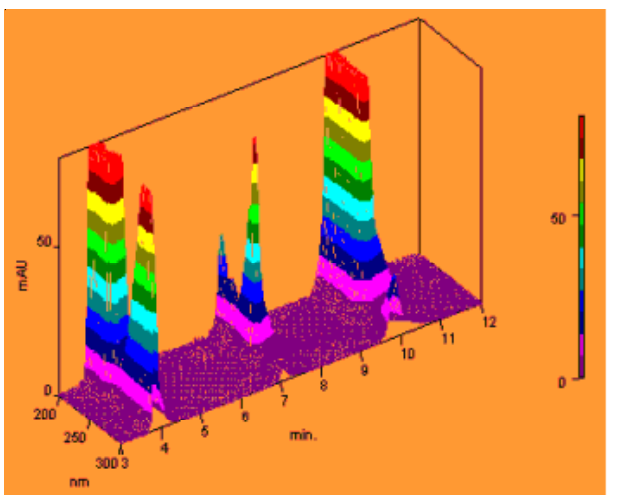

Fig. 5. Chromatograms of isomers (3-acetyl acenaphthene and 5-acetyl acenaphthene $)^{2.3}$

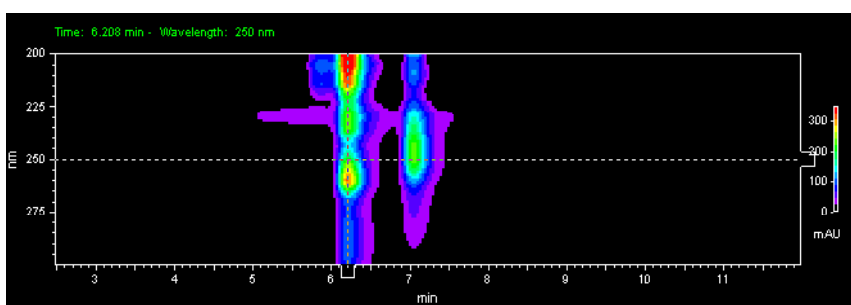

Overlaid Spectra

Whe
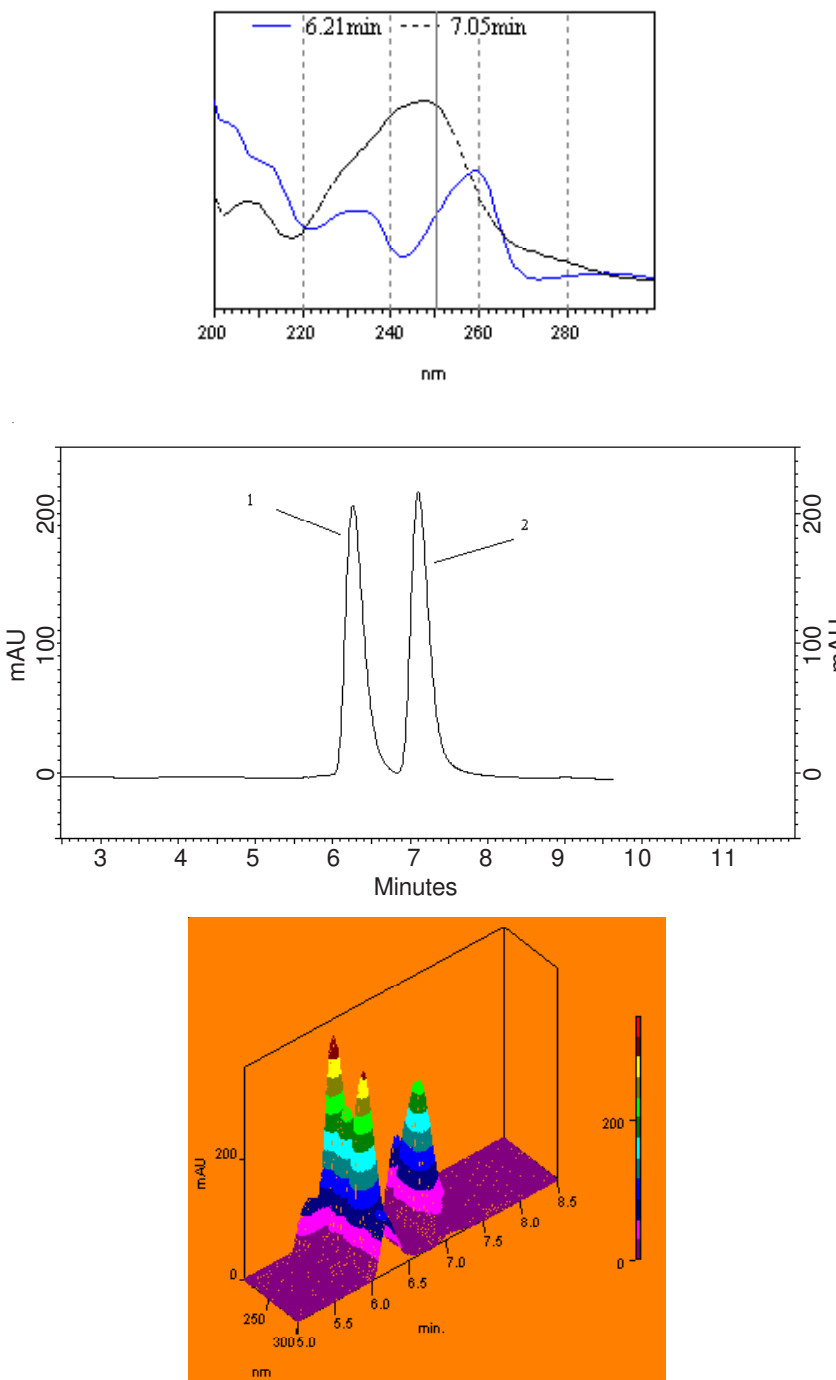

Fig. 6. Chromatograms of isomers (8-hydroxyquinoline-1-oxide and 8hydroxyquinolin-2(1H)-one)
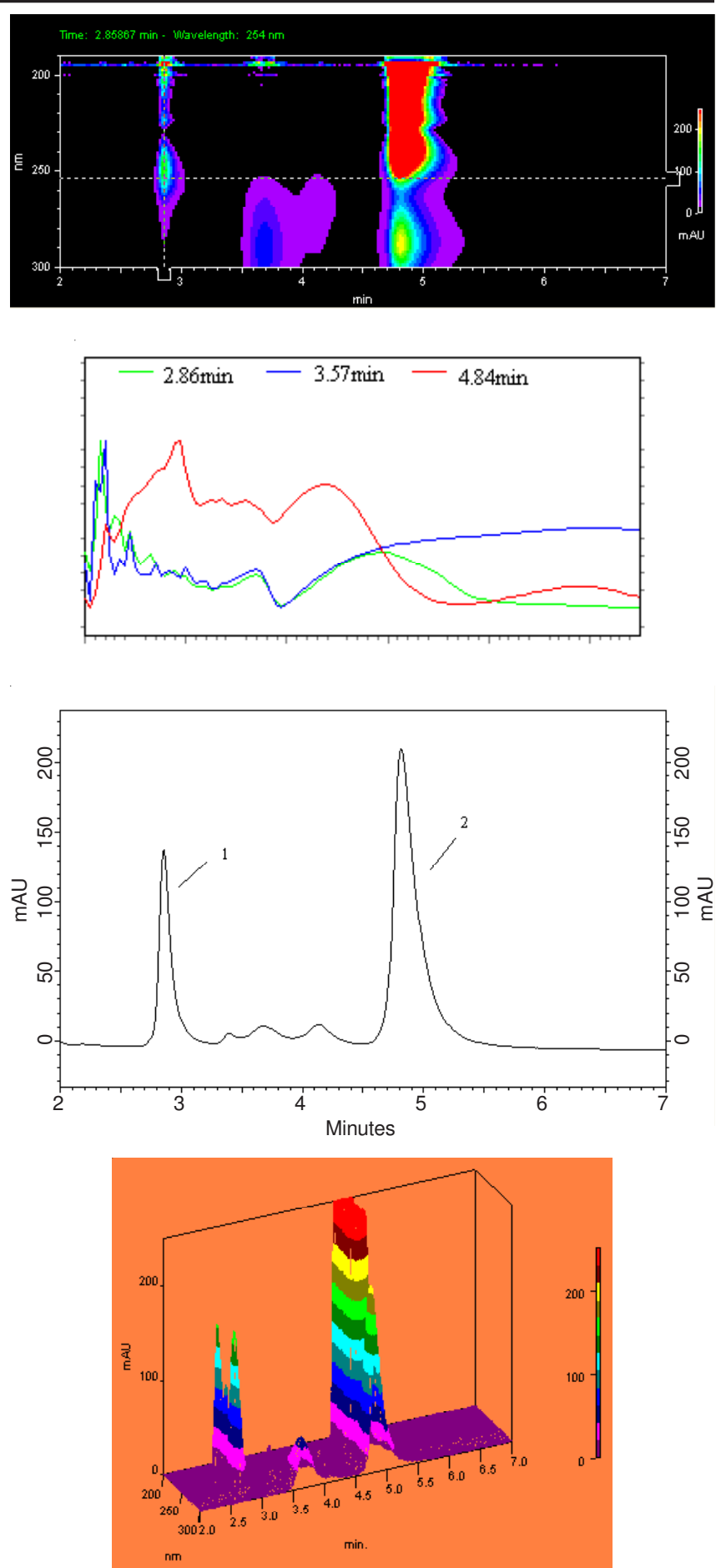

Fig. 7. Chromatograms of homologue (benzylamine and phenylethylamine)

\section{Conclusion}

By comparing the HPLC chromatograms of enantiomers, diastereomers, isomers and homologueas, we found that the three-dimensional chromatograms of enantiomers and diastereomers have considerable consistency, while the isomers and homologueas do not have this consistency. Based on the principle, a convenient approach to discriminate the peaks for chiral compounds with diode array detector is established. So it would be possible to use this approach to primarily discriminate the peaks for chiral compounds in the resolution and determination optical purity of chiral compounds. 


\section{REFERENCES}

1. R. Vespalec and P. Bocek, Chem. Rev., 100, 3715 (2000)

2. F. Gasparrini, D. Misiti and C. Villani, J. Chromatogr. A, 906, 35 (2001).

3. G. Felix, J. Chromatogr. A, 906, 171 (2001).

4. I. Brondz, D. Ekeberg, D.S. Bell, A.R. Annino, J.A. Hustad, R. Svendsen, V. Vlachos, P. Oakley, G.J. Langley, T. Mohini, C.G. Amaury and F. Mikhalitsyn, J. Pharm. Biomed. Anal., 43, 937 (2007).

5. P.S. Mukherjee, J. Pharm. Biomed. Anal., 43, 464 (2007).

6. R.A. Coe, J.O. Rathe and J.W. Lee, J. Pharm. Biomed. Anal., 42, 573 (2006).

7. R.N. Rao, D. Nagaraju and A.N. Raju, J. Pharm. Biomed. Anal., 41, 766 (2006)

8. G. Gubitz and M.G. Schmid, Biopharm. Drug Dispos., 22, 291 (2001).

9. G. Torok, L. Goetelen, R. Luyckx and P.V. Broeck, J. Pharm. Biomed. Anal., 39, 425 (2005).

10. M. Maftouh, C. Granier-Loyaux, E. Chavana, J. Marini, A. Pradines, Y.V. Heyden and C. Picard, J. Chromatogr. A, 1088, 67 (2005).

11. D. Mangelings, N. Hardies, M. Maftouh, C. Suteu, D.L. Massart and Y.V. Heyden, Electrophoresis, 24, 2567 (2003).

12. M.M. Hefnawy, M.A. Sultan and M.M. Al-Shehri, J. Chromatogr. B, 856, 328 (2007).
13. Y.W. Zhang, S. Yao, H. Zeng and H. Song, Curr. Pharm. Anal., 6, 114 (2010).

14. Y.W. Zhang, D. Yang, S. Yao, Y. Cao and H. Song, J. Chromatogr. Sci., 49, 67 (2011)

15. S.P. Li, P. Li, C.M. Lai, Y.X. Gong, K.K.W. Kan, T.T.X. Dong, K.W.K. Tsim, Y.T. Wang, J. Chromatogr. A, 1036, 239 (2004).

16. M. Pelillo, M. Bonoli, B. Biguzzi, A. Bendini, T.G. Toschi and G. Lercker, Food Chem., 87, 465 (2004).

17. B. Tavazzi, R. Vagnozzi and D.D. Pierro, Anal. Biochem., 277, 104 (2000).

18. A. Bogomolov and M. McBrien, Anal. Chim. Acta, 490, 41 (2003).

19. Q. Sun, H. Song and C.J. Shen, Chin. J. Anal. Chem., 34, 1179 (2006).

20. Y.W. Zhang, D. Yang, Y.M. Xie, Q.S. Liu, X. Wang and H. Song, Chin. J. Anal. Lab., 28, 72 (2009).

21. X. Xu, H Song, J. Sun, C. Fu and S.J. Shen, Chin. J. Anal. Lab., 26, 89 (2007).

22. D. Yang, X. Xu, Y.J. Chen, L. Zhang, C.H. Yao and H. Song, Chin. J. Anal. Lab., 27, 52 (2008)

23. C. Xu, W.F. Luo, L.J. Li, S. Yao and H. Song, Asian J. Chem., 22, 3923 (2010). 